\title{
Mapping the Kinematics of the Narrow-Line Region in the Seyfert Galaxy NGC $4151^{1}$
}

\author{
V. Das ${ }^{2}$, D.M. Crenshaw ${ }^{2,3}$, J.B. Hutchings ${ }^{4}$, R.P. Deo ${ }^{2,5}$, S.B. Kraemer $^{6}$, T.R. Gull ${ }^{7}$, M.E. \\ Kaiser $^{8}$, C.H. Nelson ${ }^{9}$, and D. Weistrop ${ }^{10}$
}

\begin{abstract}
Using The Hubble Space Telescope's Space Telescope Imaging Spectrograph (HST's $S T I S)$, observations of the [O III] emission from the narrow-line region (NLR) of NGC 4151 were obtained and radial velocities determined. Five orbits of HST time were used to obtain spectra at five parallel slit configurations, at a position angle of $58^{\circ}$, with spatial resolution $0^{\prime \prime} .2$ across and $0^{\prime \prime} .1$ along each slit. A spectral resolving power $(\Delta \lambda / \lambda)$ of $\sim 9,000$ with the G430M grating gave velocity measurements accurate to $\sim 34 \mathrm{~km} \mathrm{~s}^{-1}$. A kinematic model was generated to match the radial velocities, for comparison to previous kinematic models of biconical radial outflow developed for low-dispersion spectra at two slit positions. The new high-resolution spectra permit the measurement of accurate velocity dispersions for each radial-velocity component. The full-width at half-maximum (FWHM) reaches a maximum of $1000 \mathrm{~km} \mathrm{~s}^{-1}$ near the nucleus, and generally decreases with increasing distance to about $100 \mathrm{~km} \mathrm{~s}^{-1}$ in the
\end{abstract}

\footnotetext{
${ }^{1}$ Based on observations made with the NASA/ESA Hubble Space Telescope. STScI is operated bt the Association of Universities for Research in Astronomy, Inc., under NASA contract NAS5-26555. These observations are associated with proposal GTO-8473

${ }^{2}$ Department of Physics and Astronomy, Georgia State University, Astronomy Offices, One Park Place South SE, Suite 700, Atlanta, GA 30303, das@chara.gsu.edu

${ }^{3}$ crenshaw@chara.gsu.edu

${ }^{4}$ Dominion Astrophysical Observatory, National Research Council of Canada, Herzberg Institute of Astrophysics, 5071 West Saanich Rd., Victoria, BC V9E 2E7, Canada, john.hutchings@nrc-cnrc.gc.ca

${ }^{5}$ deo@chara.gsu.edu

${ }^{6}$ Catholic University of America and Laboratory for Astronomy and Solar Physics, NASA's Goddard Space Flight Center, Code 681, Greenbelt, MD 20771, stiskraemer@yancey.gsfc.nasa.gov.

${ }^{7}$ Laboratory for Astronomy and Solar Physics, NASA's Goddard Space Flight Center, Code 681, Greenbelt, MD 20771, theodore.r.gull@nasa.gov

${ }^{8}$ Department of Physics and Astronomy, John Hopkins University, 3400 North Charles Street, Baltimore, MD 21218-2695, kaiser@pha.jhu.edu

${ }^{9}$ Department of Physics and Astronomy, Drake University, Des Moines, IA 50311, charles.nelson@drake.edu

${ }^{10}$ Department of Physics, University of Nevada, Las Vegas, 4505 Maryland Pkwy., Las Vegas, NV 89154-4002, weistrop@physics.unlv.edu
} 
extended narrow-line region (ENLR), starting at about 6 " from the nucleus. In addition to the bright emission knots, which generally fit our model, there are faint high velocity clouds which do not fit the biconical outflow pattern of our kinematic model. These faint clouds occur at the turnover points of the outflowing bright clouds. We suggest possible scenarios that could explain these rogue clouds: (1) backflow resulting from shocks and (2) outflow outside of the bicones, although the latter does not explain how the knots are ionized and accelerated. A comparison of our observations with a high-resolution radio map shows that there is no evidence that the kinematics of the NLR clouds are affected by the radio lobes that comprise the inner jet.

Subject headings: galaxies: kinematics and dynamics-galaxies: individual (NGC 4151) galaxies: Seyfert-AGN: emission lines-ultraviolet: galaxies

\section{Introduction}

Seyfert galaxies are generally classified into two groups, based on their spectra and emission line widths in the optical. Seyfert 1 galaxies are characterized by broad permitted lines with typical FWHM velocities $\geq 1000 \mathrm{~km} \mathrm{~s}^{-1}$, and narrower forbidden lines with FWHM $\approx 500 \mathrm{~km} \mathrm{~s}^{-1}$, with a non-stellar underlying continua. The spectra of Seyfert 2 galaxies show only the narrow emission lines, and their non-stellar contribution is normally detected only in polarized light. The generally accepted unification scheme states that the two classes of galaxies are not disparate, but that Seyfert 2 galaxies are Seyfert 1s with nuclei that are obscured by a dusty molecular torus. Antonucci \& Miller (1985) discovered broad Balmer and FeII lines in the Seyfert 2 galaxy NGC 1068. From polarization arguments they concluded that the lines must be emanating from regions synonymous with the broad-line region (BLR) of Seyfert 1s. Subsequent studies reinforced the notion that Seyfert 2 BLR are obscured from the line of sight but can be observed via scattered radiation (Antonucci 1993). Therefore it is now generally accepted that the Seyfert designation 1 or 2 is contingent upon the orientation angle as perceived by the observer.

A more dynamical analysis is needed to determine if these two types of active galactic nuclei (AGN) are indeed similar and powered by the same processes. Kinematic studies of the narrow line region (NLR) can provide insights into the intricate dynamical forces occurring within its vicinity. With the STIS spectrograph aboard HST, such studies can be easily accomplished efficiently. High spatial and spectral resolution data in particular can provide us with detailed diagnostics of the NLR and its vicinity. We have therefore undertaken an investigation of the kinematics across the NLR of NGC 4151, one of the nearest Seyfert galaxies.

This type of observation and kinematic modelling was previously done on NGC 1068, a bright Seyfert 2 galaxy (Crenshaw \& Kraemer 2000a). The data were taken with the low resolution $(\Delta \lambda / \lambda$ $=1000)$ G430L grating of STIS, and the radial velocity measurements were made using [OIII $] \lambda 5007$ 
emission along two slit positions $0^{\prime \prime} .1$ wide. They found that a simple biconical outflow model closely matched the observed radial velocities such that emission-line knots accelerate out from the nucleus to $\sim 1000 \mathrm{~km} \mathrm{~s}^{-1}$ then decelerate back to the systemic velocity. The same model was applied to NGC 4151 (Crenshaw \& Kraemer 2000b, hereafter Paper 1), based on STIS long-slit low resolution and slitless medium resolution spectra (Hutchings et al. 1999; Kaiser et al. 2000; Nelson et al. 2000). In Paper 1 they pointed out that the observed velocity field can be well matched by tilting the model bicone by about $40^{\circ}$ out of the plane of the sky, as opposed to only $5^{\circ}$ for NGC 1068 .

In this paper, we present the same modeling procedures as in the two previous studies, but at a spectral resolving power of 9000, and five parallel slit positions covering the NLR of NGC 4151. This provides the most detailed map of the kinematics in a Seyfert 1 NLR, similar to that obtained for the Seyfert 2 galaxy NGC 1068 (Cecil et al. 2002). We used a systemic velocity of $997 \mathrm{~km}$ $\mathrm{s}^{-1}$, based on H I observations of the outer regions of the host galaxy (Pedlar et al. 1992) and a Hubble constant of $75 \mathrm{~km} \mathrm{~s}^{-1} \mathrm{Mpc}^{-1}$, which gives us a distance of $13.3 \mathrm{Mpc}$ to NGC 4151, with $0^{\prime \prime} .1$ corresponding to a projected linear scale of $6.4 \mathrm{pc}$ on the sky.

\section{Observations and Analysis}

The STIS observations were taken on 2000 July 02 over five orbits (proposal ID 8473, PI: J. Hutchings) with the HST/STIS CCD through a slit of $52^{\prime \prime} \times 0^{\prime \prime} .2$, and dispersed with the mediumresolution G430M grating, whose spectral resolving power is $\sim 9000$. The STIS image scale is $0^{\prime \prime} .05 / \mathrm{pix}$ and the spatial resolution is $0^{\prime \prime} .1 / \mathrm{pix}$ in the cross-dispersion direction. The five orbits yielded spectra for five parallel slit positions, each at a position angle of $57.8^{\circ}$. There were three exposures per slit with a total of 40 minutes of integration for slit 1 and 48 minutes for the remaining slits. Figure 1 shows the slit placements and orientation superimposed on a WFPC2 [O III] emission-line image of NGC 4151 showing the inner $4^{\prime \prime}$. Slit 1 was centered on the optical continuum nucleus and the remaining slits were placed at $0^{\prime \prime} .2$ and $0^{\prime \prime} .4$ on either side, parallel to slit 1 . The image was taken with the [O III] filter, which has a central wavelength of 4961, and a bandpass of 4820-5100 $\AA$, which includes the $\mathrm{H} \beta$ line and the [O III] $\lambda \lambda$ 4959, 5007 redshifted emission lines. Most of the emission comes from within $2^{\prime \prime}$ of the bright central knot, although [O III] emission can be traced as far as $6^{\prime \prime}$ on either side.

Figure 2 shows a fully reduced STIS image of the two [O III] lines as they appeared through slit 1 . The bright horizontal line through the image is the continuum emission produced by the nucleus. The top of the image corresponds to the northeast direction and the horizontal scale is in wavelength. Both [O III] lines clearly show an overall trend in that the majority of clouds in the upper region are receding or redshifted and those clouds in the bottom part of the image are approaching or blueshifted. Close to the nucleus, within $\sim 2^{\prime \prime}$, the clouds appear highly dispersed.

We used the IDL software developed at NASA's Goddard Space Flight Center for the STIS Instrument Definition Team to reduce the spectra. Cosmic-ray hits were identified and removed from observations by comparing the three images obtained during each orbit. Hot or warm pixels were replaced by interpolation in the dispersion direction. Wavelength calibration exposures obtained 
after each observation were used to correct the wavelength scale for zero-point shift. The spectra were geometrically rectified and flux-calibrated to produce a constant wavelength along each slit column and fluxes in units of ergs $\mathrm{s}^{-1} \mathrm{~cm}^{-2} \AA^{-1}$ per cross dispersion pixel.

Each spectral image produced one spectrum per cross-dispersion pixel along the slit, and centered around the two bright doppler-shifted [O III] emission lines. We used the brighter of the two emission lines ( $\lambda 5007)$ and fitted each [O III] component with a local continuum and Gaussians. Noisy spectra roughly $\geq 6^{\prime \prime}$ from either side of the nucleus were not fit. Close to the nucleus, and on either side, emission knots showed two and sometimes three major kinematic components (Figure 3), and in such cases we fitted each identifiable peak with Gaussians.

Figure 3 depicts a progression of spectra illustrating how the components fluctuate with position. The graphs represent spectra taken from slit 1 and range in distance from $0^{\prime \prime} .3$ to $0^{\prime \prime} .55$ in increments of $0^{\prime \prime} .05$, going away from the nucleus in the northeast direction. In (a), there are two distinct kinematic components of emission, which we fit with two Gaussians. Going from (a) to (b), a third component emerges. A possible fourth component emerges in (c), but was not measured by our fitting routine. The second component shrinks in (c), and disappears in (d), while the fourth component is now detected and labeled ' 2 '. This new second component grows stronger while the third shrinks and disappears from (d) through (f). The appearance of the same components in multiple rows along the slit indicates that we are obtaining multiple measurements of spatially-resolved knots, the brightest of which can be seen in Figure 1. For comparison purposes, we separated these components by relative intensities and plotted them in different colors (later in the paper).

The central peaks of the Gaussians give us the central wavelengths from which we used the Doppler formula to find radial velocities for each component. There are three sources of uncertainty in the velocity measurements. The first results from the fact that the [O III] emission lines are not Gaussians as shown in Figure 3. By actually measuring the centroid of a line for a number a spectra, we found that our Gaussian fitting routine introduced a maximum fitting error of $0.75 \AA$ in the centroid. The second error comes from emission cloud displacements from the center of the $0^{\prime \prime} .2$ slit in the dispersion direction. We calculated that a $0^{\prime \prime} .1$ offset would contribute an error of $0.56 \AA$. Noisy spectra introduced the third error. Various noisy spectra was measured several times and the wavelength shifts were found to fluctuate by $0.30 \AA$ maximum. The errors were converted to velocities and added in quadrature to produce a total maximum error of $\pm 60 \mathrm{~km} \mathrm{~s}^{-1}$. We also varied the continuum placement for randomly selected spectra but found that this did not shift the central wavelength significantly.

The S/N ratio of the integrated [O III] flux was large near the nucleus, approaching values of 1000 , and then decreased rapidly to $\sim 100$ at about $1^{\prime \prime}$ from the nucleus. The $\mathrm{S} / \mathrm{N}$ ratio reduced to $\sim 50$ at $3^{\prime \prime}, \sim 30$ at $4^{\prime \prime}$, and $\lesssim 10$ at $6^{\prime \prime}$. We did not attempt to measure the emission if the $\mathrm{S} / \mathrm{N}$ ratio was $<3$, which typically occured at distances of $>6^{\prime \prime}$. This trend was seen in all five slits.

In Paper 1, mostly bright clouds with one or two kinematic components were detected and fitted to a model. In this paper, our data show multiple components (1, 2, and 3$)$ and hence in order to make a comparison to Paper 1, we separated these kinematic components according to total flux in the line. We assigned the color red to components with the strongest flux; blue, to components 
with the the next strongest flux, and black, to components with the weakest flux. This kind of separation of the components by fluxes incorporates a minor problem. Consider Figure 4 showing a progression of spectra from (a)-(f). The components are ordered 1, 2, and 3 going from left to right, and the colors that we assign are marked below each component. Notice that in (b), the second component is labeled black (since it has less flux under the line) and the third component is labeled blue. For similar reasons, the black and blue colors switched places again in (c), as a new second component emerged. The third component shrinked in (d), and disappeared in (e). The relative nature of our separation criteria is made clear in (e)-(f); with only two components detected, they are labeled red and blue, and when we have detected only one component, we labeled those red. In our velocity plots, this results in occasional mixing between the low and medium flux clouds, but does not significantly affect our model, since it was based on a general fit for all points. The plot of fluxes (bottom plot) in Figures 5 and 6 clearly show the effects of misidentification are small; as one can see the colors are clearly separated.

Figures 5 and 6 show the kinematic components in plots of radial velocities, FWHM, and fluxes as a function of projected position from the nucleus. Negative positions corresponds to the southwest direction. Note that the points tend to clump together, which reflects the ability of STIS to resolve the clouds. In the upper plots, the brightest emission line clouds at each position in the northeast are red shifted, while those in the southwest are blue shifted relative to the host galaxy. The highest velocities occur closer to the nucleus and velocities as high as $800 \mathrm{~km} \mathrm{~s}^{-1}$ can be seen in approach and recession. The general trend is an increase in radial velocity from near zero at the nucleus to a maximum redshift or blueshift within $\sim 1^{\prime \prime}$ of the nucleus. Between $\sim 2^{\prime \prime}$ to $\sim 4^{\prime \prime}$ the velocities reduce from about $200 \mathrm{~km} \mathrm{~s}^{-1}$ to $100 \mathrm{~km} \mathrm{~s}^{-1}$ and at distances of about $4^{\prime \prime}$ or more from the nucleus, the radial velocities taper off to the ENLR rotational velocities. The medium flux clouds generally show the same pattern as the bright ones except in slits 1 and 4 where they are not confined to only blue shifts in the SW. Also in slit 2, the medium flux clouds show blueshifts in the NE. The low flux clouds also follow similar patterns as the bright clouds except in slits 1 and 4 where one can see additional flow patterns opposite to that of the bright clouds. The FWHM in the middle plots show a range of widths at each position, but a decreasing average width with distance from the nucleus at all flux levels. In the bottom plot, all three components show a strong decrease in flux with distance and significant structure that corresponds to the emission line knots.

Hutchings et al. (1999) found a number of low-flux, high velocity clouds in NGC 4151 using a combination of WFPC2 and STIS images and STIS slitless spectra. Some of these faint clouds did not fit into the general picture of biconical outflow (e.g. redshifts were detected in the SW where blueshifts dominate). To check for consistency, we identified Hutchings et al.'s clouds in our data and compared our velocities with theirs. Our measurements are closely correlated over a wide range of velocites as shown in Figure 7. Also shown on top of the figure are the letters of various high velocity knots, labeled according to Hutchings' scheme. The positions of Knots E and J in Hutchings et al.'s image should have placed them in our slits 1 and 5 respectively, but they were not found in our data. On average, the difference between Hutchings et al.'s velocity and ours is $134 \mathrm{~km}$ $\mathrm{s}^{-1}$, but there is no significant systemic offset. In addition to these knots, we found many more high 
velocity low and medium emission knots. These can be seen in the velocity plots of Figures 5 and 6 .

\section{Kinematic Models and Results}

In Crenshaw \& Kraemer (2000a), the radial outflow from the Seyfert 2 galaxy, NGC 1068 was accounted for by a simple biconical radial velocity law, $v=k r, r$ being the distance from the central source and $k$ being a constant. They used STIS low-resolution long-slit spectra taken in the [O III] emission regime. The model bicone was evacuated along its axis up to an inner opening angle of $26^{\circ}$ from which it was assumed to have a filling factor of 1 up to an outer opening angle of $40^{\circ}$. The gas within the filled region accelerates from rest close to the nucleus to about $1300 \mathrm{~km} \mathrm{~s}^{-1}$, at a distance 140 pc away. From this point it decelerates back to systemic velocity at a distance of about $300 \mathrm{pc}$ from the nucleus. The deceleration of the gas followed a law with the velocity decreasing linearly with distance, $v=v_{\max }-k^{\prime} r$. The best-fit model used an inclination of $5^{\circ}$ (relative to the plane of the sky) for the bicone axis. In Paper 1, the same modeling procedures were used to map the kinematics of NGC 4151. The bicones were assumed to have identical geometrical and physical properties and a filling factor of 1 within the cones and no absorption of the [OIII] photons. Interestingly, the velocity laws $v=k r$ and $v=v_{\max }-k^{\prime} r$ further out also worked in this model. The middle column of Table 1 shows the parameters for the best fit model from Paper 1 .

In this paper we test the model in Paper 1 using higher resolution data and more coverage of the NLR of NGC 4151. The kinematic modelling code used in Paper 1 was updated to test different velocity laws, corresponding to radial outflow. Parameters for the bicone that were allowed to vary in our code include the extent of each cone along its axis $\left(z_{\max }\right)$, the minimum and maximum half opening angles $\left(\theta_{\text {inner }}\right.$ and $\left.\theta_{\text {outer }}\right)$, the inclination of its axis out of the plane of the sky $\left(i_{\text {axis }}\right)$, the velocity turnover radius $\left(r_{t}\right)$ (measured radially outward from the nucleus), and the maximum velocity at the turnover point $\left(v_{\max }\right)$. All the above parameters were assumed to be the same for both top (northeast) and bottom (southwest) parts of the bicone. We tested various velocity laws for the outflow and found that two of them fit the data reasonably well. The first law was $v=k r$ and $v=v_{\max }-k^{\prime} r$ for the acceleration and deceleration phases of the gas respectively. The second law was $v=k \sqrt{r}$ and $v=v_{\max }-k^{\prime} \sqrt{r}$ for the two phases. We referred to these laws as the $r$ law and the $\sqrt{r}$ law respectively ${ }^{1}$. A constant velocity law and velocity proportional to higher powers of radius did not work well. Motion perpendicular to the bicone axis and infall do not work (see Paper 1).

Our code generates a two-dimensional velocity map and samples this map with slit positions, orientations, and widths that match those of the observations. Figure 8 shows our model velocity field projected onto the sky. The purple color is the highest negative velocity and the red is the

\footnotetext{
${ }^{1}$ Note that the latter corresponds to constant acceleration with radius and not the former, as stated in some of our earlier papers. The acceleration in the papers was proportional to radius. This can be seen by solving the simple differential equation $a=\frac{d v}{d t}=v \frac{d v}{d r}=r^{n}$, where $\mathrm{n}=1$ for the $r$ law, and $\mathrm{n}=0$ for the $\sqrt{r} l a w$.
} 
highest positive velocity, with intermediate velocities given in gradients. Next to the fields are velocity extractions from slit 1 done to visually aid the reader. The extraction consists of four parts, two from the back side of the bicone, and two from the front side. The upper two graphs consists of velocities extracted from the top and bottom parts respectively, of the back side of the bicone. Similarly the lower two graphs represent the front side of the bicone. In each graph, the relevant extracted section is in shaded grey, in addition to the total extraction from slit 1, which put things into perspective. Radial velocities were extracted along the five slits and plotted as a function of position in arc seconds. Note that the model was tilted out of the plane of the sky so that the extracted radial velocities would best match the general trend of the data. The other bicone parameters were then adjusted until the best match between model and data was established. In this fit, emphasis was placed on the high and medium flux points. The low flux points in slits one and four indicate a flow pattern which in some cases is opposite to that determined from the model; for the rest of the slits however, there is general agreement.

Figure 9 shows all five slits with the models in shaded grey and the observed data points in colors. Zero position corresponds to the nucleus. These radial velocity plots show that the northeast side is mostly redshifted, and the southwest side is mostly blueshifted, except for some low luminosity clouds (black points), and a few medium luminosity clouds (blue points), which exhibit an unusual flow pattern. These are shown by the arrows in Figure 9 and to some extent, between $0^{\prime \prime}$ and $-2^{\prime \prime}$ in Figure 9 (e). Plots (a) through (e) represent models with the $r$ law and correspond to slits 1 through 5 respectively. Plot (f) shows a fit for slit one with the $\sqrt{r}$ law. A closer inspection of plots (a) and (f) shows minuscule differences in the fit, with (a) being slightly better. Minor tweaking of the parameters would result in good agreement for the $\sqrt{r}$ law in slit 1 , however the $r$ law tends to be more consistent across all five slits.

The final model parameters for NGC 4151 are presented in Table 1. For comparison, the fitting parameters from Paper 1 are also presented. Following the deceleration trend of the knots in all five slits, the model suggests that the knots return to systemic velocity at a larger distance from the nucleus than previously found in Paper 1. This extends the bicone longer than in Paper 1, to $400 \mathrm{pc}$ as opposed to $288 \mathrm{pc}$. The turnover point is much closer in, $96 \mathrm{pc}$ compared to $162 \mathrm{pc}$ and our inner opening angle is $15^{\circ}$, which makes our model less evacuated along the bicone axis. The high luminosity knots seem to travel outward from the nucleus at lower inclination and was modeled in Paper 1 as such; however, in this paper we also incorporate medium and low luminosity knots into the plots and hence our match suggests a larger inclination of $45^{\circ}$. The other parameters, inner and outer opening angles, and maximun velocity of the gas, show only minor differences between the two papers. The maximum velocity before turnover was increased in our paper to account for the fast moving medium and low luminosity clouds seen in Figure 9. These clouds are accelerated to high speeds close to the nucleus and they therefore play a major role in modelling the turnover point, which we found to be $\sim 96$ pc. If we had used only the bright clouds (red points) then our turnover point would have been larger and similar to that of Paper 1.

Figure 10 shows a plot of FWHM versus radial velocity for all five slits combined. There seems to be no definite correlation between the FWHM and the absolute value of the radial velocity. This 
indicates that the directions of velocity dispersion are not preferentially along the direction of the velocity vector. The FWHM is therefore not due to a purely radial gradient. However, various low luminosity clouds do show some trend. Figure 11 shows evidence of a correlation for a combined plot of isolated groups of points shown by the arrows in Figure 9. These groups of points do not follow the general trend of the bright clouds and they consist of only medium and low flux points. This leads us to believe that these particular knots are either preferentially disturbed in the direction of the velocity vector, or that they exhibit turbulence in proportion to their velocity. Note that the faintest clouds have higher FWHM and radial velocity. We will look at these rogue clouds more carefully later in the paper.

Figure 12 shows a model of NGC 4151 with the bicone as it should appear in the sky to us, using our final model parameters. The position angle of the Galaxy is $22^{\circ}$, with an inclination of $20^{\circ}$ to the line of sight, the SW side being closer to us. The position angle of the bicone is $60^{\circ}$, and its inclination is $45^{\circ}$. The maximum half opening angle of the bicone is shown here to be $33^{\circ}$. Note that our line of sight is outside of the bicone, at an angle of $12^{\circ}$ with respect to the nearest part of the bicone. The $\beta$ angle, which is the angle between the bicone axis and the normal to the galactic disk, is $36^{\circ}$. Due to the bicone's large opening angle, it will intersect a sufficiently thick galactic disk (e.g., a scale height of $\sim 100 \mathrm{pc}$ ). The NE bicone will also intersect the NE galactic disk due to symmetry. This intersection produces the observed geometry of the ENLR as shown by Evans et al. (1993).

\section{Interaction with Radio Jet}

The kinematics of the [O III] clouds in some Seyfert galaxies have been interpreted as outflow away from (perpendicular to) the jet axis. Several authors have suggested close relationships between the radio jets and the optical emission such that hot gas around the radio lobes expands and propels the NLR clouds to high velocities (see Gallimore et al. 1996; Capetti et al. 1997b: Axon et al. 1998). Although this scenario seemed plausible when applied to NGC 1068, it failed to explain the turnover velocity where emission knots started to decelerate back to systemic velocity, at about $2^{\prime \prime}$ from the nucleus (Crenshaw \& Kraemer 2000a). Also the clumpy nature of the radio knots could not explain the orderly large-scale flow pattern of the radial velocities. The axis outflow was discussed in Nelson el al. (2000) in relation to NGC 4151. They found that regardless of inclination, the radial velocities should appear both red-shifted and blue-shifted with equal magnitudes at each position along the axis. However NGC 4151 has mostly blue-shifted clouds in the southwest region and mostly red- shifted clouds in northeast of the nucleus. Therefore, radial outflow matches the data much better.

To test whether the radio knots are responsible for locally disturbing the [O III] clouds, we generated plots from radio maps taken by the Very Long Baseline Array plus the Very Large Array (see Mundell el al. 2003, and references therein) together with radial velocity plots from our data, and compared them within the $2^{\prime \prime}$ range that the radio data cover. We extracted radio emission 
along the same slit positions as our 5 slit positions in Figure 1. The radio fluxes were summed across each $0^{\prime \prime} .2$ slit width and normalized to the peak of the brightest radio knot, which was in slit 1. Radio flux maxima in slits 3 through 5 were only about one tenth the value as those in slits 1 and 2 .

Figure 13 shows our slits overlaid on top of the radio map. The radio jet is not parallel with the [O III] axis, but lies close to one edge of the projected bicone. Slits 1 and 2 contained the two brightest radio knots and the entire image spans about $4^{\prime \prime}$. Figure 14 shows intensity plots of the radio emission together with the our radial velocities. Here we expect that bright radio knots would produce roughly symmetric red and blue shifts at the positions of the knots if the axis outflow applies to NGC 4151. However we see no evidence for such a scenario in any slit. Figure 14 (b) shows some blue shifted clouds at the position of the radio knot, but no corresponding red shifted clouds are observed. We see some red-shifted material in the upper bicone at $0^{\prime \prime} .5$, but this is too far away to be affected by the radio knot. Near the brightest radio flux in (c) and (d), we see some blue and red shifted [O III] clouds respectively, but again there are no symmetric counterparts for these clouds. Similar plots of the velocity widths show no evidence for larger FWHM at the positions of the radio lobes. We conclude that the radio lobes have no discernible effects on the kinematics of the NLR clouds.

\section{Discussion}

We confirm our previous biconical outflow models (Crenshaw \& Kraemer 2000b), that were used to match the radial velocities of the NLR in NGC 4151, using higher velocity-resolution STIS spectra. In Crenshaw \& Kraemer (2000b), the model was based on two slit positions at different position angles. Here we use five parallel slits, all at the same position angle, but different from that used in Crenshaw \& Kraemer (2000b). However, the previous model was found to be consistent with ours, except for a few adjustments. The turnover point of the velocity (where clouds start to decelerate) occurs much closer to the nucleus than the previous model showed, and the point where the clouds velocity approaches systemic velocity is further away. These two differences follow from the fact that we were able to detect fainter, high-velocity knots closer in to the nucleus. We note that some of the high-velocity low-flux components do not match the model as shown by the arrows in Figure 9. These high-velocity clouds had been detected in Hutchings et al. (1999) to have velocities of both approach and recession on both sides of the nucleus. We see similar trends in Figure 9 (a), (b), (d), and somewhat in (e). The rest of the low-flux clouds are in agreement with the model.

The sum of the half opening angle and the inclination of the axis puts the near-sided southwest cone at an angle of $14^{\circ}$ with respect to the line of sight for Paper 1 and $12^{\circ}$ for this paper. This means that we are still looking outside the bicone, contrary to the Seyfert 1 classification,

and general Seyfert unification models. The most likely explanation for an unobscured view of the nucleus is that the bicone edges, and therefore the torus edges, are not sharp.

The radio jet lobes do not seem to cause any disturbance to the [O III] emission and are not 
directly responsible for the acceleration of the gas, as is evident from Figure 14. One should see both blue and red-shifts roughly at the same position in these plots, but there is no evidence of this. The same plots with FWHM also show no correlation between position of the radio lobes and kinematics of the [O III] knots.

We propose possible scenarios to explain the faint clouds that do not fit the biconical outflow pattern, such as in Figure 9 (a). One explanation is that of backflow due to shocks, as a result of outflowing bright clouds impacting on an ambient medium. The low luminosity clouds could be flowing back along the bicone with a velocity that is proportional to the outflow velocity; that is, the higher the outflow velocity, the higher the backflow velocity. The top arrow in Figure 9 (a) points to rogue points which may have resulted from fast outflowing gas in the near side of the southwest bicone, with the low emission gas moving in the opposite direction of general outflow, leaving a trail of clouds in the direction of the nucleus; see location b in Figure 15. Because the near side of the cone has a higher projected velocity, we see the rogue clouds trailing back along this side. The bright clouds are accelerated in the first few tenths of an arcsec and the rogue points mimic the same behaviour, but in the opposite direction. The same argument holds for clouds pointed at by the second arrow in Figure 9 (a). This backflow most likely comes from the far side of the cone ( $\mathrm{d}$ in Figure 15), since the velocities and position there agree with those of the rogue clouds. The knots pointed at by the arrow in Figure 9 (b) can be associated with backflows from the near side of the NE bicone where their positions coincide with those of the bright clouds at location a in Figure 15. Clouds pointed at by the lower arrow in Figure 9 (d) can also be explained by backflow from the near side of the NE bicone. The velocities and positions of the rogue clouds are similar to those of the clouds in this region. The argument loses some credibility, however, when we consider the upper arrow in Figure 9 (d). These high velocity rogue knots seemed to be geometrically associated with the near side region of the SW bicone, (b in Figure 15), but there are no bright clouds in this region to contribute to the backflow behaviour.

There is another possibility which can explain the unusual flow pattern of all the rogue clouds. The argument is that the rogue clouds are moving in an entirely different geometry flowing away from the nucleus as shown in Figure 15, locations e, f, g, and h. Note that backflows from a, b, $\mathrm{c}$, and $\mathrm{d}$, are equivalent to projected velocities from $\mathrm{g}, \mathrm{f}, \mathrm{e}$, and $\mathrm{h}$, respectively. This geometrical equivalent of the backflows is but one of the ways in which the projected velocities agree, since the geometry could be positioned anywhere along the line of sight to yield the same projected velocities. We adopt the geometry whereby clouds emanate from the central nucleus, as this scenario seems to be the most plausible one. If that is the case then the flows from locations e and $\mathrm{f}$ would be at a rather large angle (near 90 degrees) with respect to the bicone axis. This would suggest that clouds are flowing close to the plane of the torus, which might explain their low flux. The problem with this scenario is that there is no means for the clouds to be ionized unless radiation is coming from holes in the torus. It is also not clear how these clouds would be accelerated without radiation or winds from the nucleus, as these clouds do indeed attain high velocities.

We prefer the first scenario, because the rogue clouds appear at the positions of the turnover velocities of the bright clouds. This supports the idea (Paper 1) that the bright clouds may be 
plunging into an ambient medium at the turnover point which cause the clouds to slow down and eventually return to zero velocity, at the same time producing the faint clouds in backflow. The second scenario seems less likely, because there is no other evidence at present for a leaky torus.

We thank C. Mundel for providing the radio map of NGC 4151 used in this study. The observations for this research were obtained as part of a NASA Guaranteed Time Observer allocation to the STIS Science Team. 


\section{REFERENCES}

Antonucci, R. 1993, ARA\&A, 31, 473

Antonucci, R. R. J., \& Miller, J. S. 1985, ApJ, 297, 621

Axon, D. J., Marconi, A., Capetti, A., Macchetto, F. D., Schreier, E., \& Robinson, A. 1998, ApJ, 496,75

Capetti, A., Macchetto, F. D., \& Lattanzi, M. G. 1997b, ApJ, 476, 67

Cecil, G., Dopita, M. A., Groves, B., Wilson, A. S., Ferruit, P., Pécontal, E., \& Binette, L. 2002, ApJ, 568, 627

Crenshaw, D. M., \& Kraemer, S. B. 2000a, ApJ, 532, 101

Crenshaw, D. M., Kraemer, S. B., Hutchings, J.B., Bradley II, L. D., Gull, T. R., Kaiser, M. E., Nelson, C. H., Ruiz, J. R., \& Weistrop, D. 2000b, AJ, 120, 1731 (Paper I)

Evans, I. N., Tsvetanov, Z., Kriss, G. A., Ford, H. C., Caganoff, S., \& Koratkar, A. P. 1993, ApJ, 417,82

Gallimore, J. F., Baum, S. A., O’Dea, C. P., \& Pedlar, A. 1996, ApJ, 458, 136

Hutchings, J. B., Crenshaw, D. M., Danks, A. C., Gull, T. R., Kraemer, S. B., Nelson, C. H., Weistrop, D., Kaiser, M. E., \& Joseph C. L. 1999, AJ, 118, 2101

Kaiser, M. E., Bradley, L. D., Hutchings, J. B., Crenshaw, D. M., Gull, T. R., Kraemer, S. B., Nelson, C. H., Riuz, J. R., \& Weistrop, D. 2000, ApJ, 528, 260

Mundell, C. G., Wrobel, J. M., Pedlar, A., \& Gallimore, J. F. 2003, ApJ, 583, 192

Nelson, C. H., Weistrop, D., Hutchings, J. B., Crenshaw, D. M., Gull, T. R., Kaiser, M. E., Kraemer, S. B., \& Lindler, D. 2000, ApJ, 531, 257

Pedlar, A., Howley, P., Axon, D. J., \& Unger, S. W. 1992, MNRAS, 259, 369 
Fig. 1.- [O III] intensity image of the NLR of NGC 4151 showing the orientation and position of the five slits taken by HST STIS. The position angle of the slits on the sky is $58^{\circ}$.

Fig. 2.- STIS G430M fully reduced CCD spectral image for slit 1, showing the emission knots of [O III] $\lambda \lambda 4959,5007$. The nucleus is the bright continuum band across the entire image. A couple of warm pixels have not been removed by our software. Spatial extent of the image (top to bottom) is $23.7^{\prime \prime}$ and the abscissa is in wavelength.

Fig. 3.- Spectra in black and fits in color showing the multi-component gaussian fits. The plots, ordered (a)-(f) are from positions separated by $0{ }^{\prime \prime} .05$, increasing in distance from the nucleus. The green dotted curves represent the fit for each component, the red is the sum of all the green curves and the blue is the difference between spectrum, (black curve) and fitted red curve. The spectra were not yet doppler corrected for the receding motion of NGC 4151. Wavelengths are in $\AA$ on the abscissa and fluxes are in ergs $\mathrm{s}^{-1} \mathrm{~cm}^{-2} \AA^{-1}$ on the ordinate.

Fig. 4. - Cartoon showing multiple components in a progression of spectra and how we separate them according to flux. The components are assigned the colors under them after separation. In (c) there is more flux under the line, hence the color blue is assigned.

Fig. 5.- Slit 1 multi-colored plots showing the components present in the emission clouds at each position. Measurements were made using the [OIII] $\lambda 5007$ emission from the NLR of NGC 4151. The colors represent high(red), medium(blue), and low(black) flux levels respectively. Radial velocities are given relative to systemic, and fluxes are in $\operatorname{ergs~} \mathrm{s}^{-1} \mathrm{~cm}^{-2}$. All graphs share a common abscissa of position in arc-seconds (") from the nucleus. The FWHM is not corrected for instrumental resolution. Note the rogue clouds in the upper left of the velocity plot.

Fig. 6.- Plots of velocity, FWHM, and flux for slits 2-5. The colors depict the usual separation of flux levels as in Figure 5.

Fig. 7.- Comparison between our and Hutchings et al.'s velocities. We found most of their knots, except knots E and J. See Hutchings et al. (1999).

Fig. 8. - Left: Back (upper) and front (lower) sides of the radial-velocity maps generated by our $v=k r$ and $v=v_{\max }-k^{\prime} r$ models. Only the outer surface of the bicone is shown for clarity. The position angle of the bicone axis is $60^{\circ}$ in the sky, so we rotate our velocity maps clockwise by $60^{\circ}$ so that the projected kinematic axis is vertical. Deep purple, green, and dark red represent radial velocities of $-700,0$, and $+700 \mathrm{~km} \mathrm{~s}^{-1}$, respectively. The 5 slits are placed onto the front half of the bicone. Right: Next to the bicones are extracted velocity plots (in black) from slit 1, for each quadrant. The velocity plot for each quadrant is given relative to total extraction from slit 1 as a form of visual aid.

Fig. 9.- Plots showing the model (in grey) and the three components in different colors. Graphs (a), (b), and (d) show unusual flow pattern, as indicated by the arrows. Graphs (a) - (e) corresponds 
to slits $1-5$ respectively, using the $r$ law, and (f) is slit 1 , using the $\sqrt{r}$ law. Positive position $\left({ }^{\prime \prime}\right)$ is Northeast.

Fig. 10. - Plot of FWHM against absolute radial velocity showing all five slits with high flux in red, medium flux in blue, and low flux in black. No obvious correlation is seen.

Fig. 11. - Combined plots of velocity vs FWHM for knots of clouds pointed at by the arrows in Figure 9. The straight line is a linear fit, with a correlation coefficient of $r=0.55$.

Fig. 12.- Bicone and host galaxy of NGC 4151 as they should appear in the sky to us. The SE side of the host galaxy is the closer side. Also the SE bicone is closer to us. The line-of-sight is toward to reader.

Fig. 13. - Position of the slits we used for extraction from the radio image. The image was taken in 1996, March, by Mundell et al. The nucleus is at the second brightest knot in the center slit (Compare to Figure 1).

Fig. 14.- Radio intensity (smooth line) and velocity positions (crosses) for all 5 slits, corresponding to (a) - (e) respectively. The radio scale on the right side is relative to the bright radio knot shown in (a).

Fig. 15.- Possible flow patterns for the faint rogue clouds that do not fit our model. The solid lines are the bicone edges. The solid arrows represent normal outflow corresponding to the highand medium-flux clouds in the model, and the dotted and dashed arrows represent possible flow patterns for the rogue clouds. Note the line-of-sight (LOS) is to the right, and the bottom of the bicone is tilted out of the plane of the sky, corresponding to $45^{\circ}$ in our model. The rectangle in the middle of the figure represents the torus. The largest opening angle is shown here (cartoon not drawn to scale). 
Table 1. KInEMATIC MODEL OF NGC 4151

\begin{tabular}{lll}
\hline \hline Parameter & $\begin{array}{c}\text { NGC 4151 } \\
(\text { Paper 1) }\end{array}$ & $\begin{array}{l}\text { NGC 4151 } \\
\text { (This Paper) }\end{array}$ \\
\hline$z_{\text {max }}$ & $288 \mathrm{pc}$ & $400 \pm 16 \mathrm{pc}$ \\
$\theta_{\text {inner }}$ & $20^{\circ}$ & $15 \pm 2^{\circ}$ \\
$\theta_{\text {outer }}$ & $36^{\circ}$ & $33 \pm 2^{\circ}$ \\
$i_{\text {axis }}$ & $40^{\circ}(\mathrm{SW}$ is closer $)$ & $45 \pm 5^{\circ}(\mathrm{SW}$ is closer $)$ \\
$v_{\text {max }}$ & $750 \mathrm{~km} \mathrm{~s}^{-1}$ & $800 \pm 50 \mathrm{~km} \mathrm{~s}^{-1}$ \\
$r_{t}$ & $162 \mathrm{pc}$ & $96 \pm 16 \mathrm{pc}$ \\
\hline
\end{tabular}




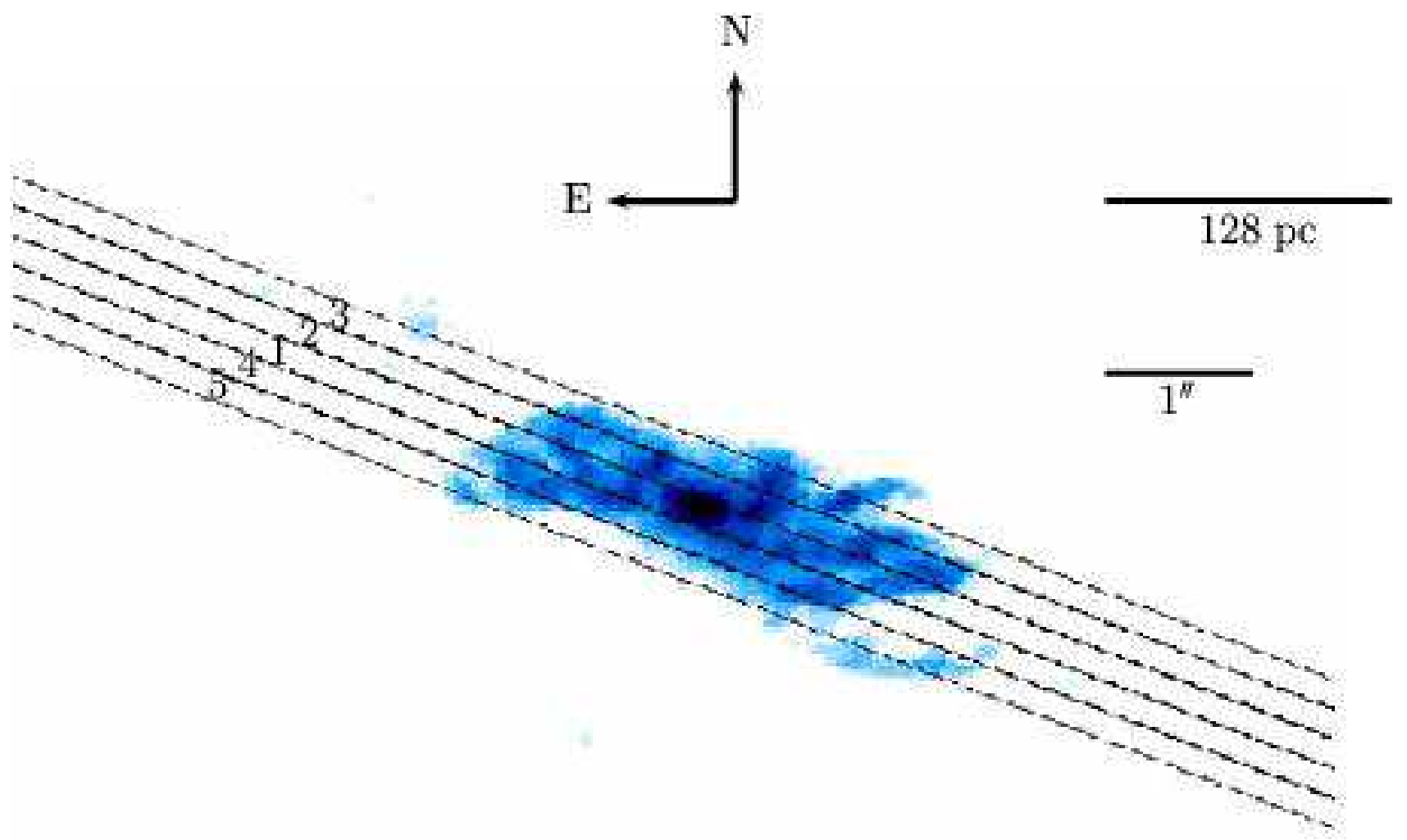

Fig. 1 


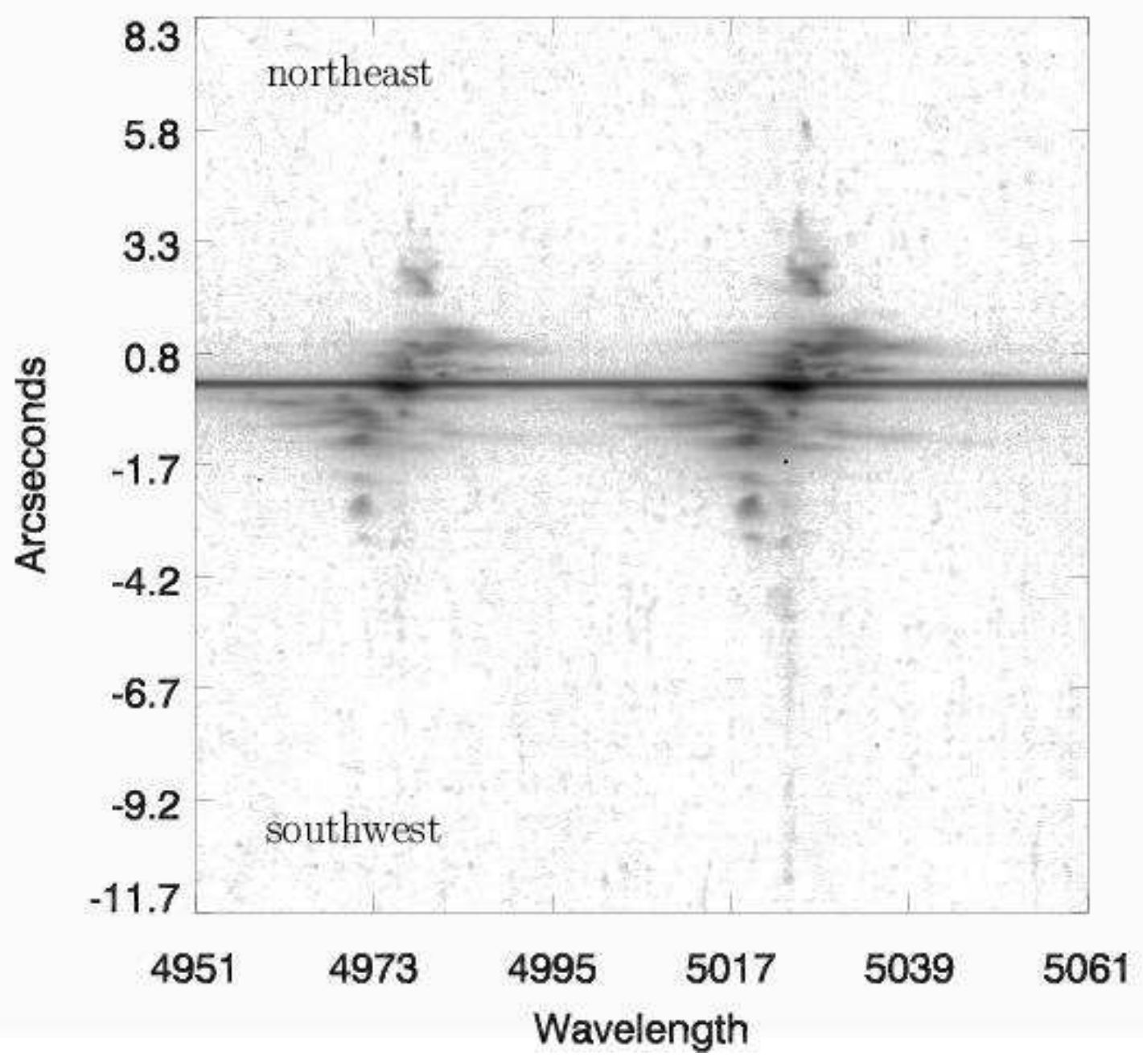

Fig. 2 
- $18-$

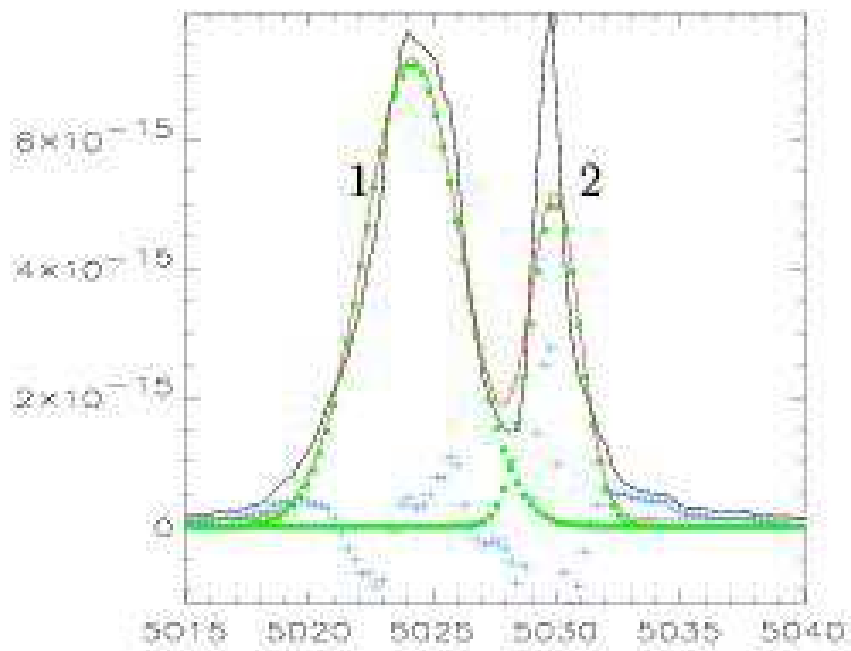

(a)

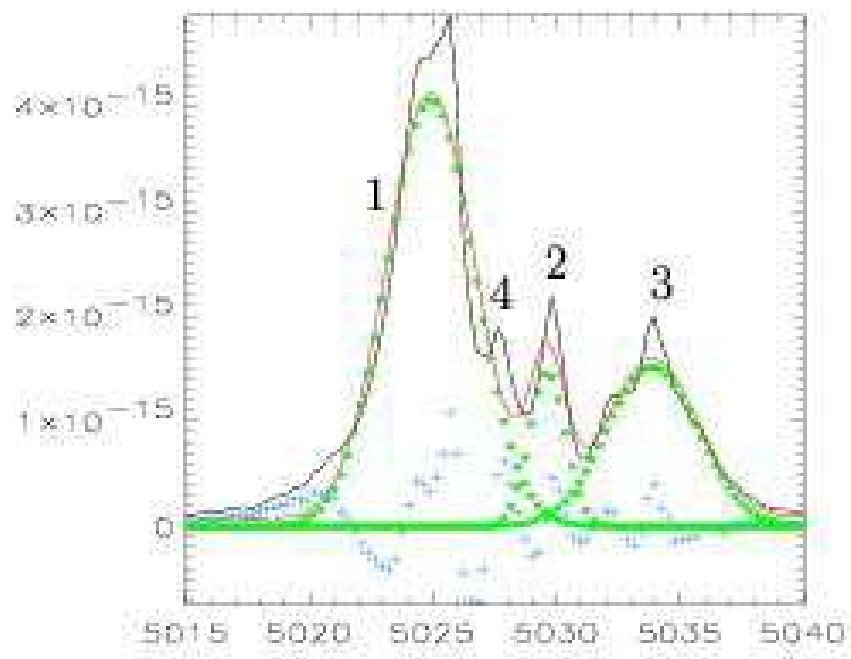

(c)

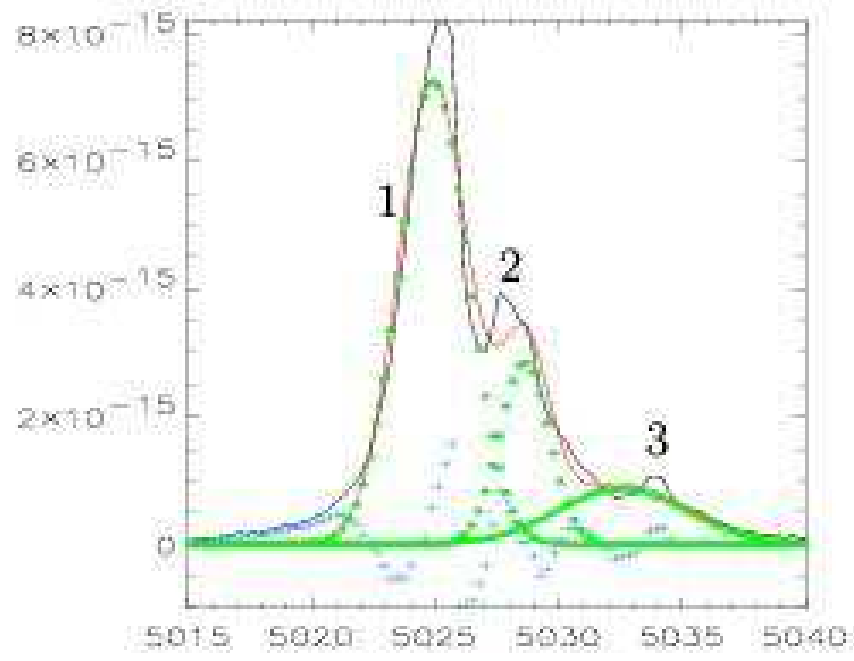

(e)

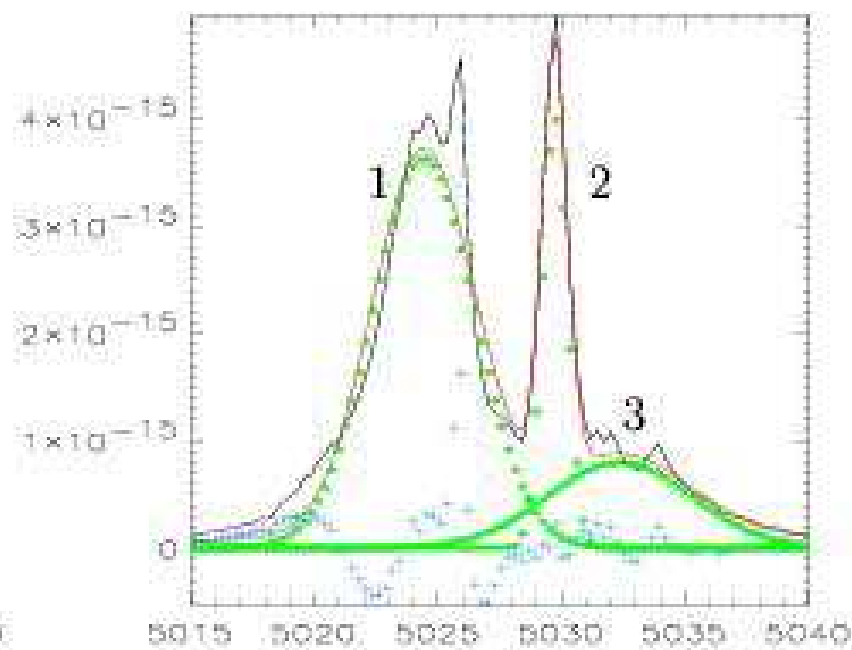

(b)

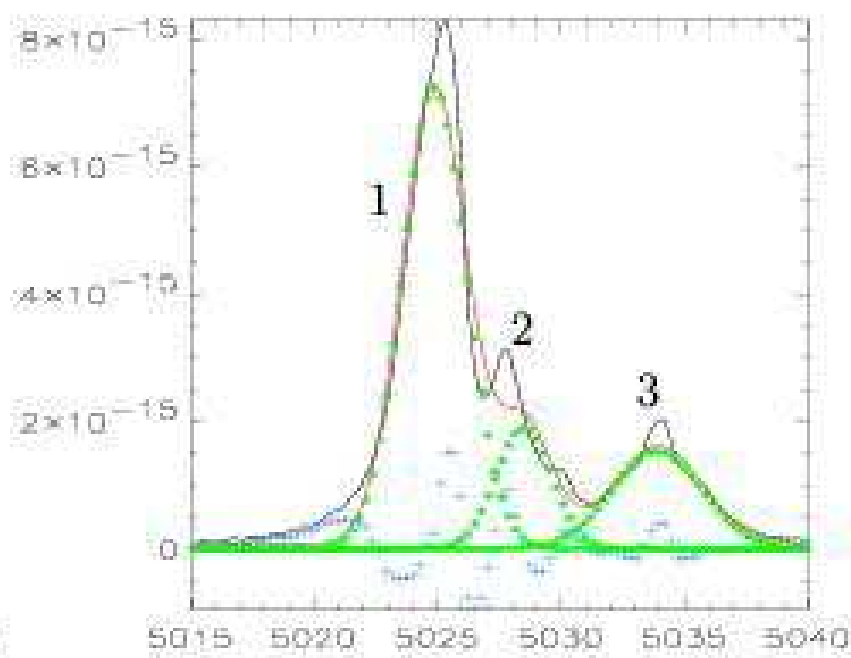

(d)

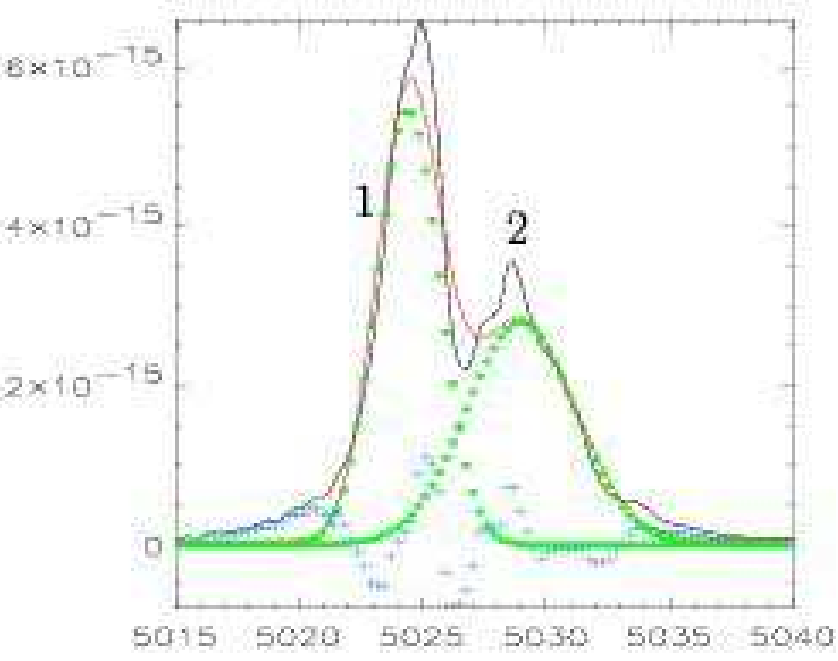

(f)

Fig. 3 


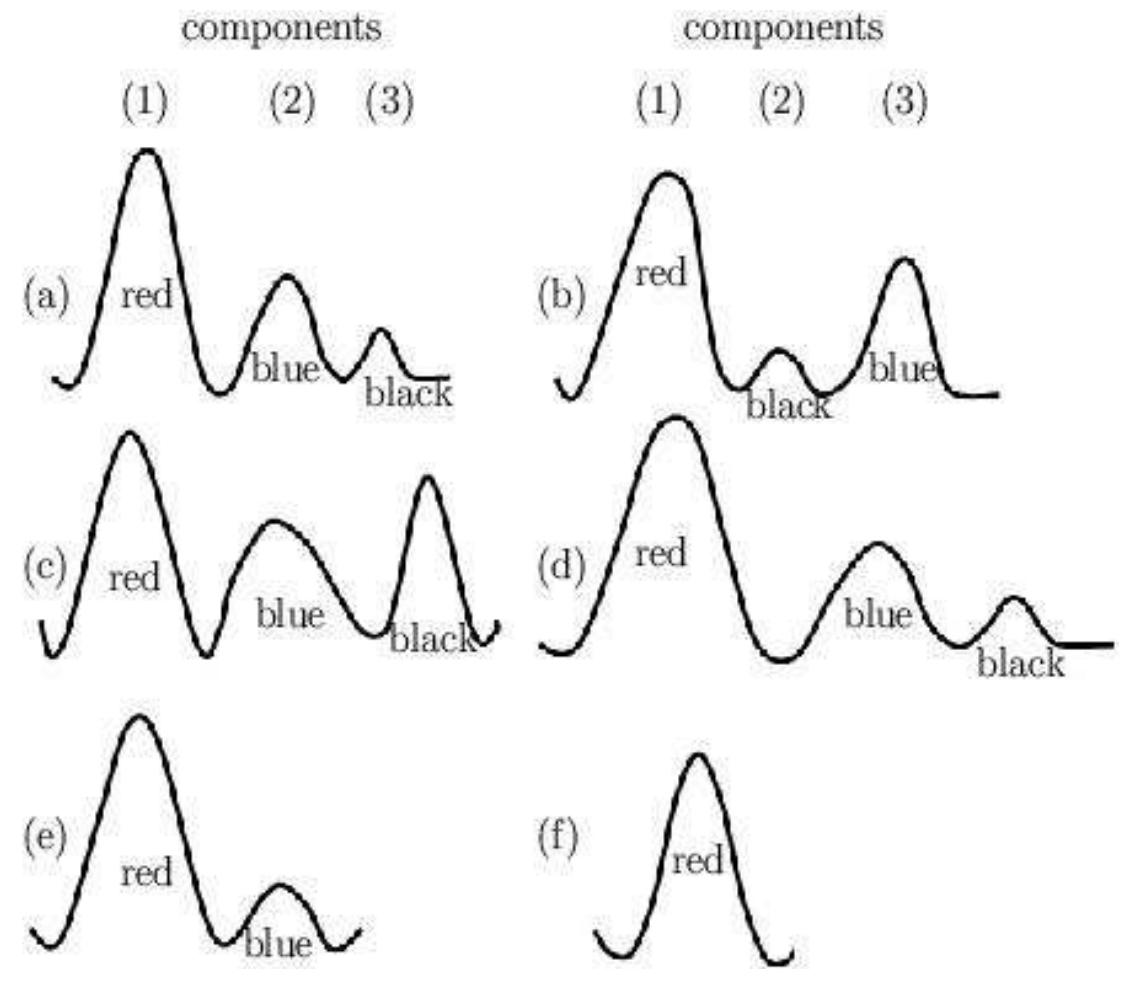

Fig. 4 


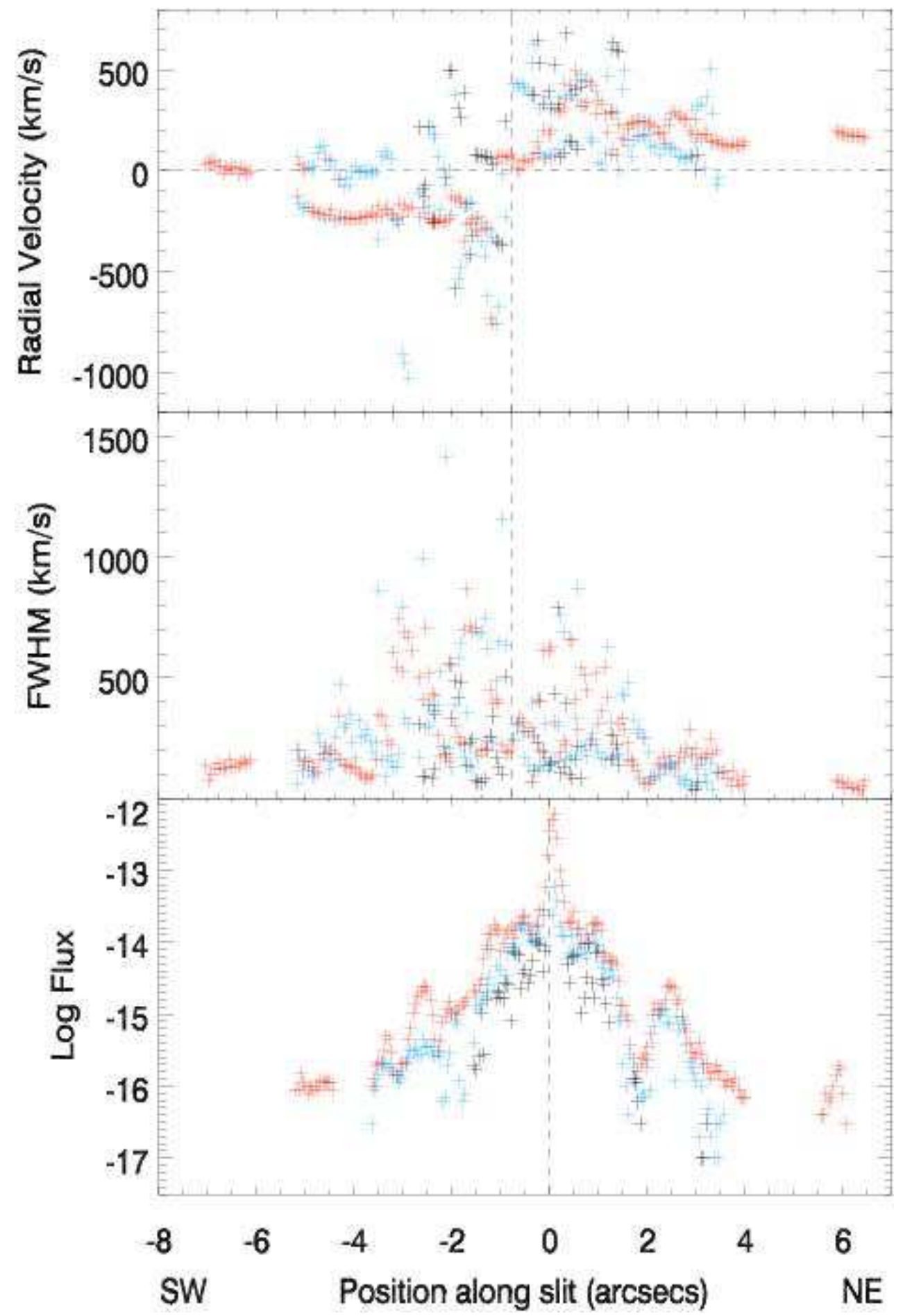

Fig. 5 

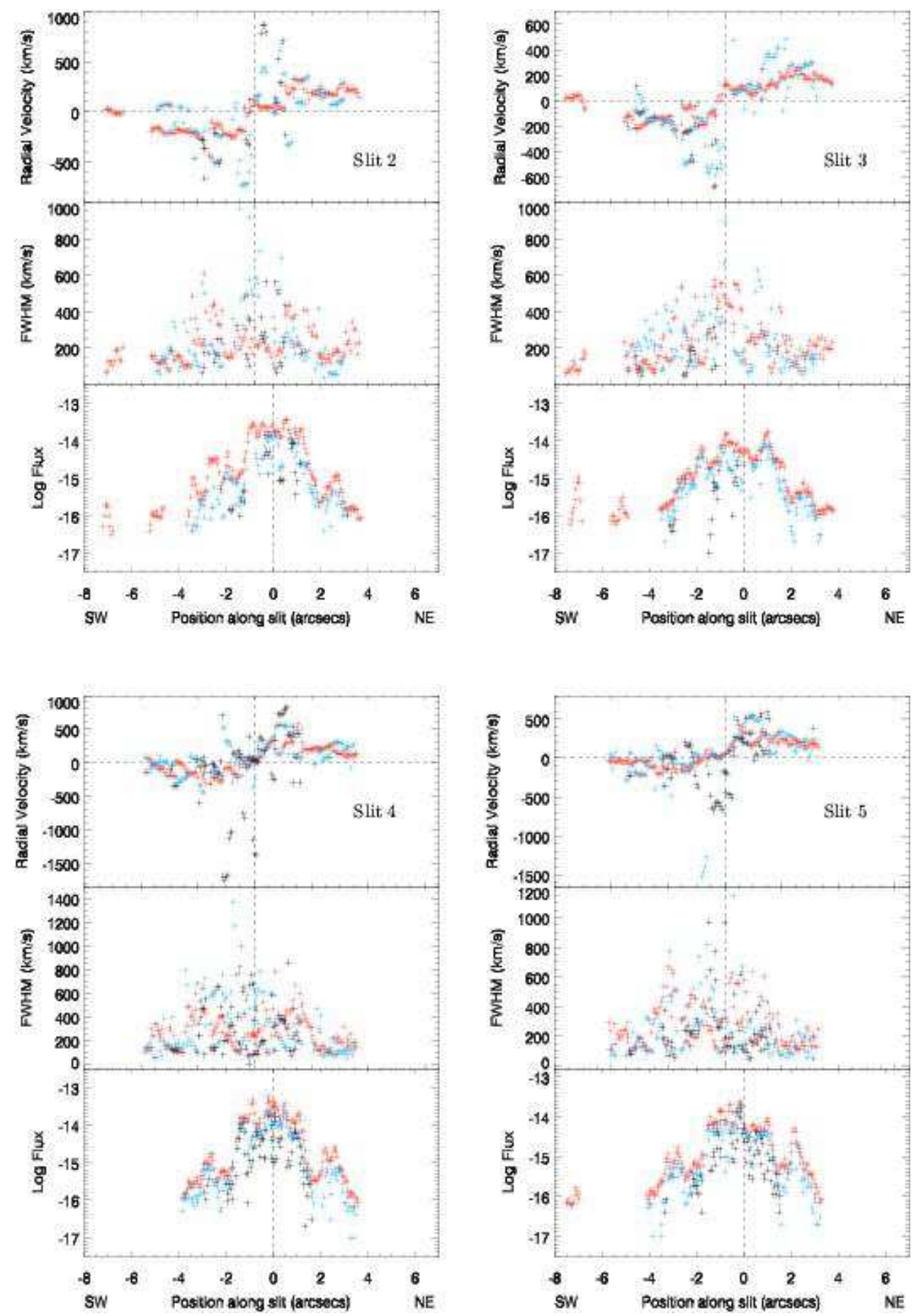

Fig. 6 


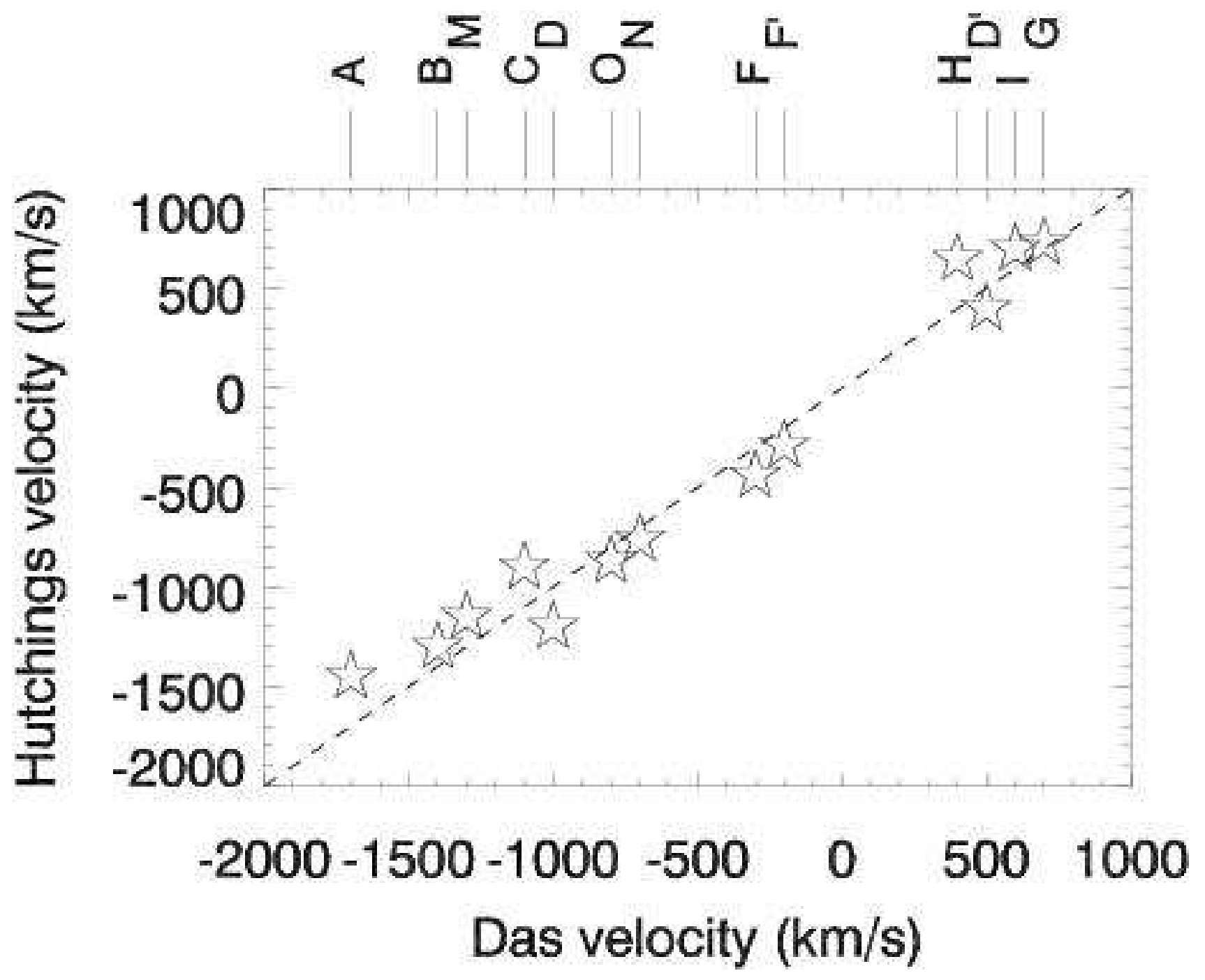

Fig. 7 

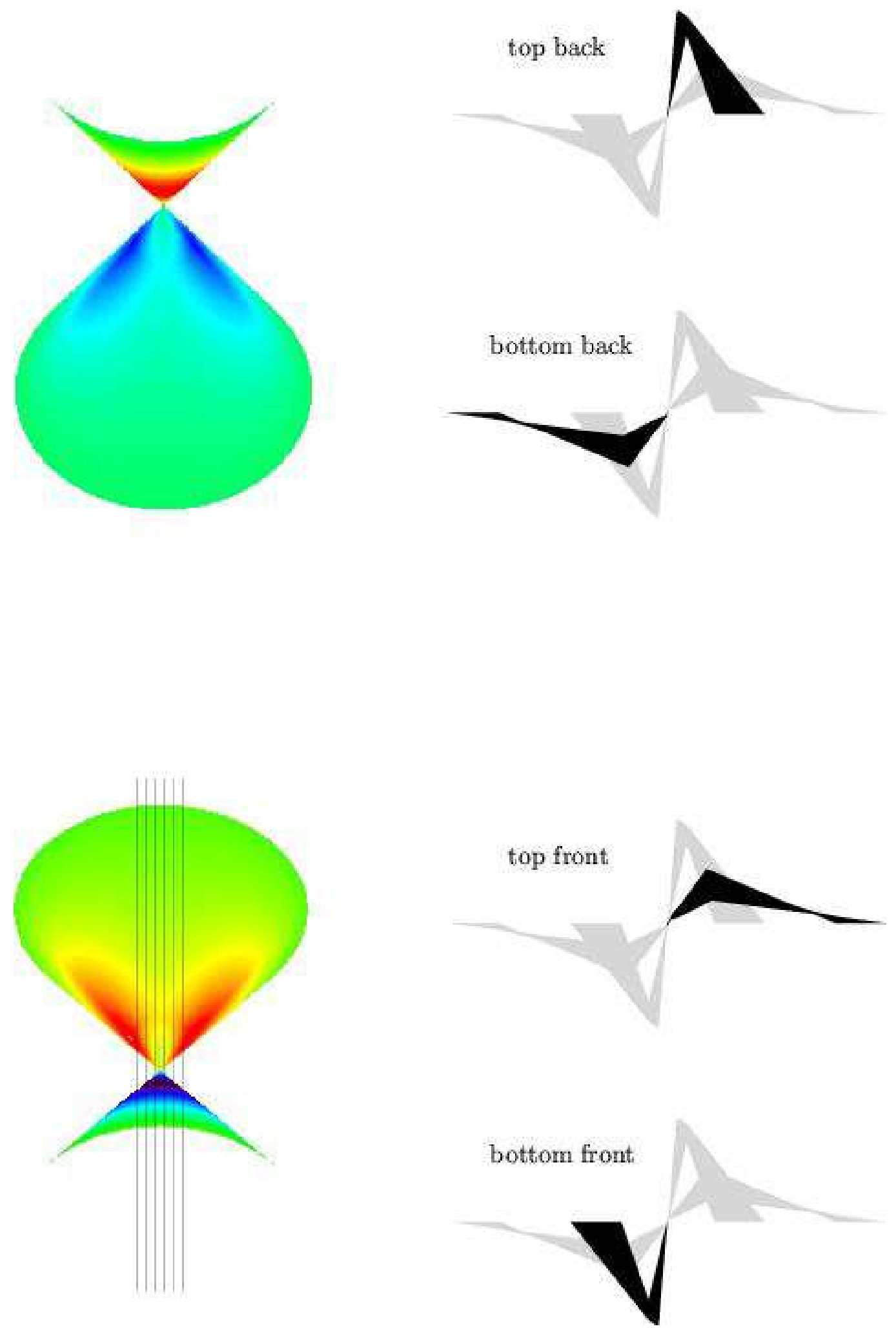

Fig. 8 

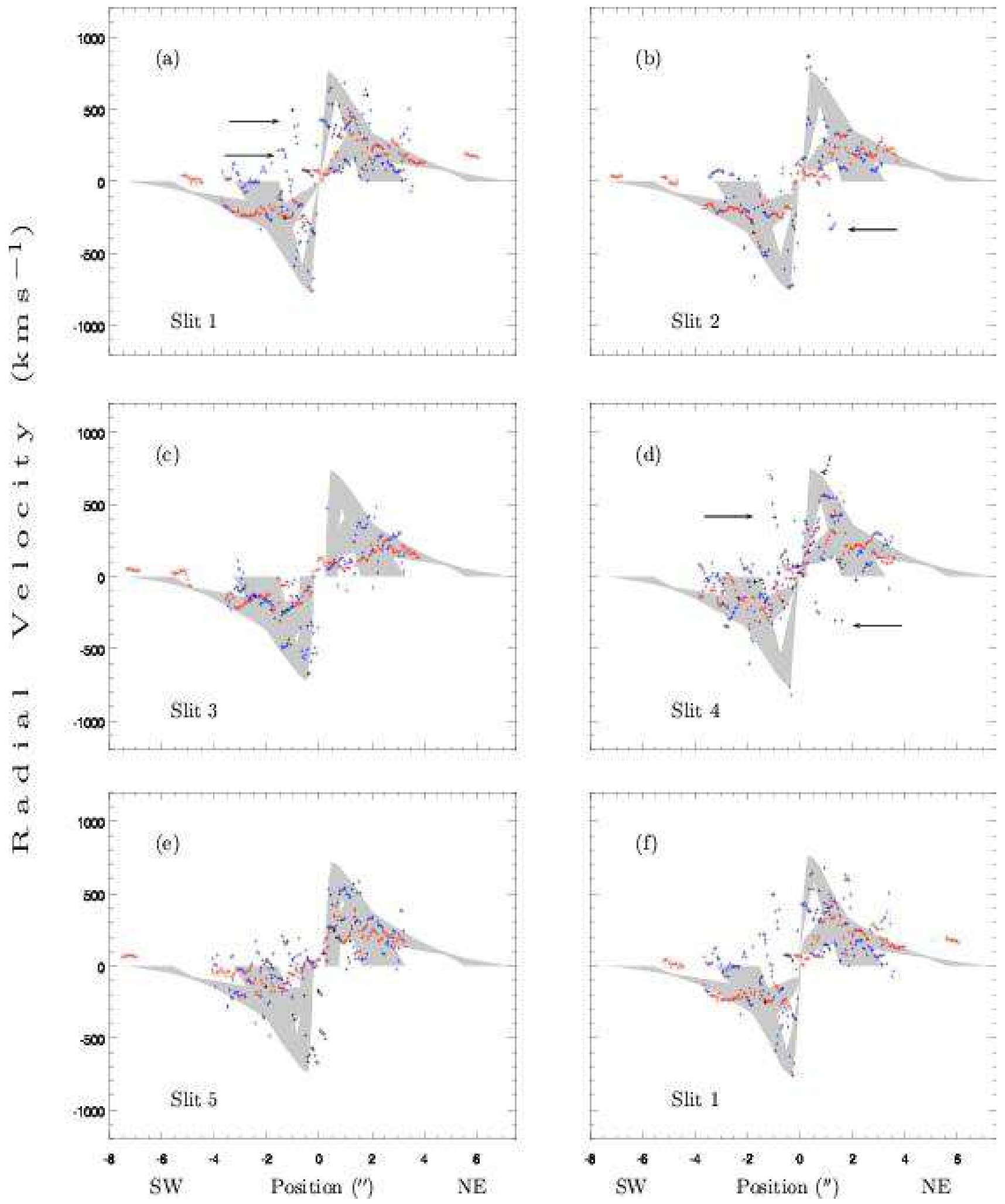

Slit 2

Slit 4

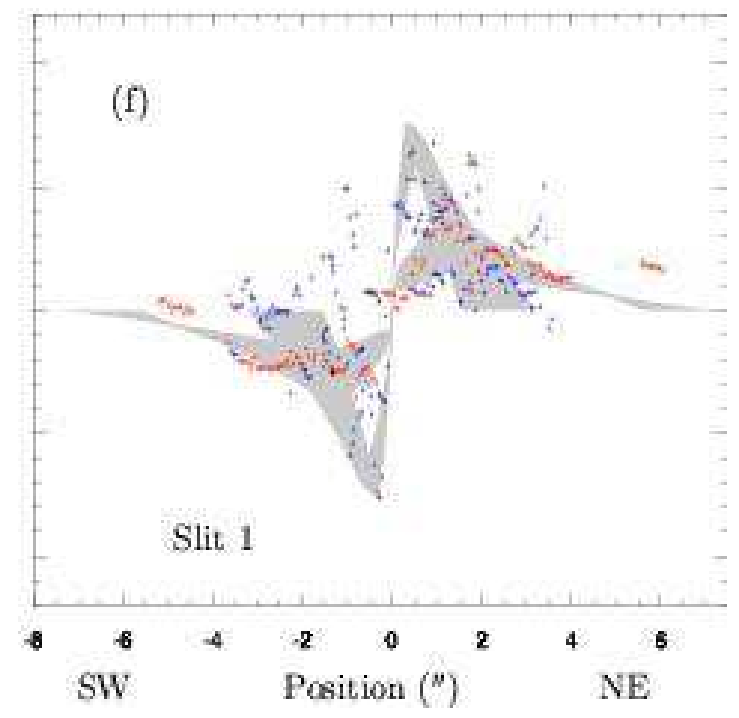

Fig. 9 


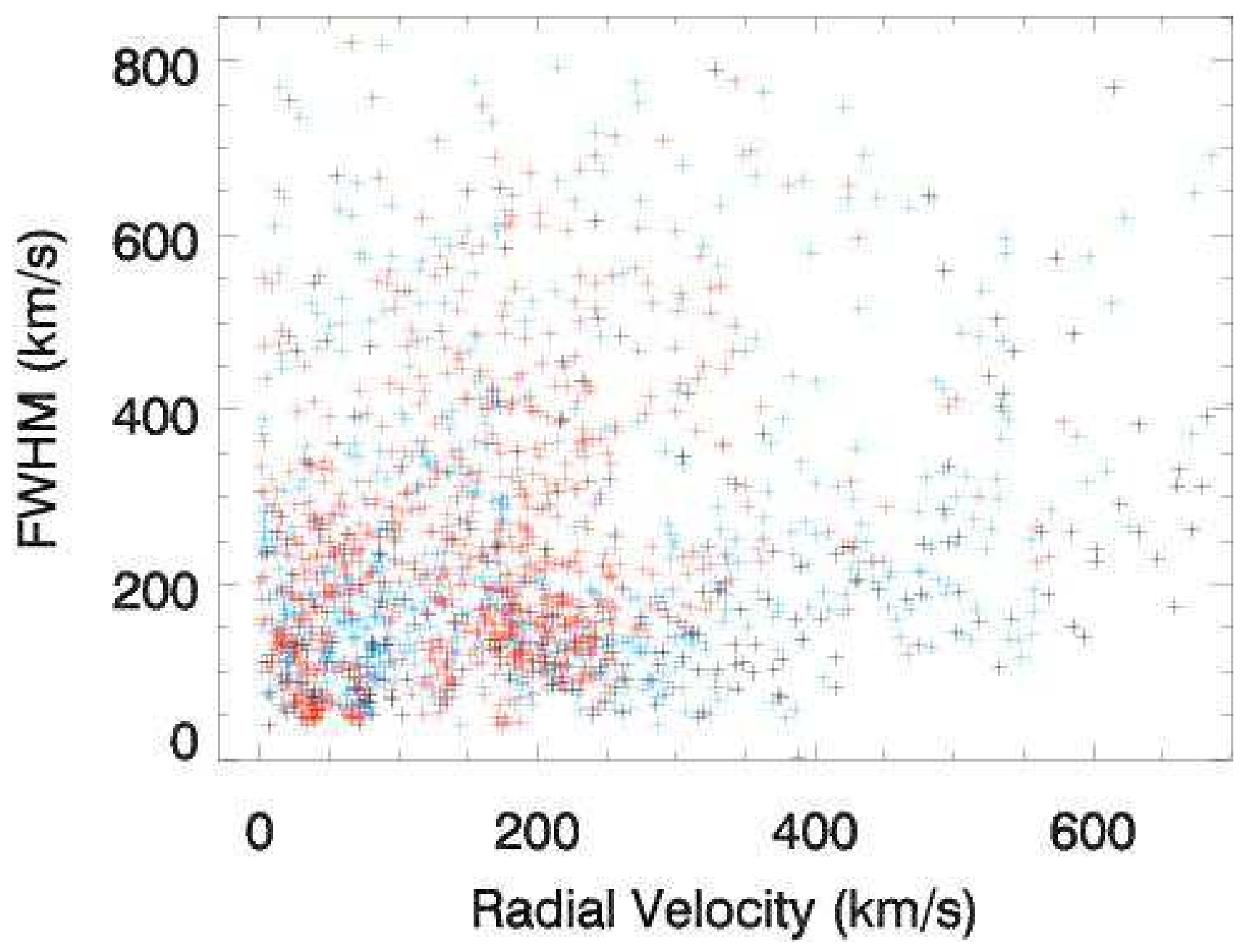

Fig. 10 


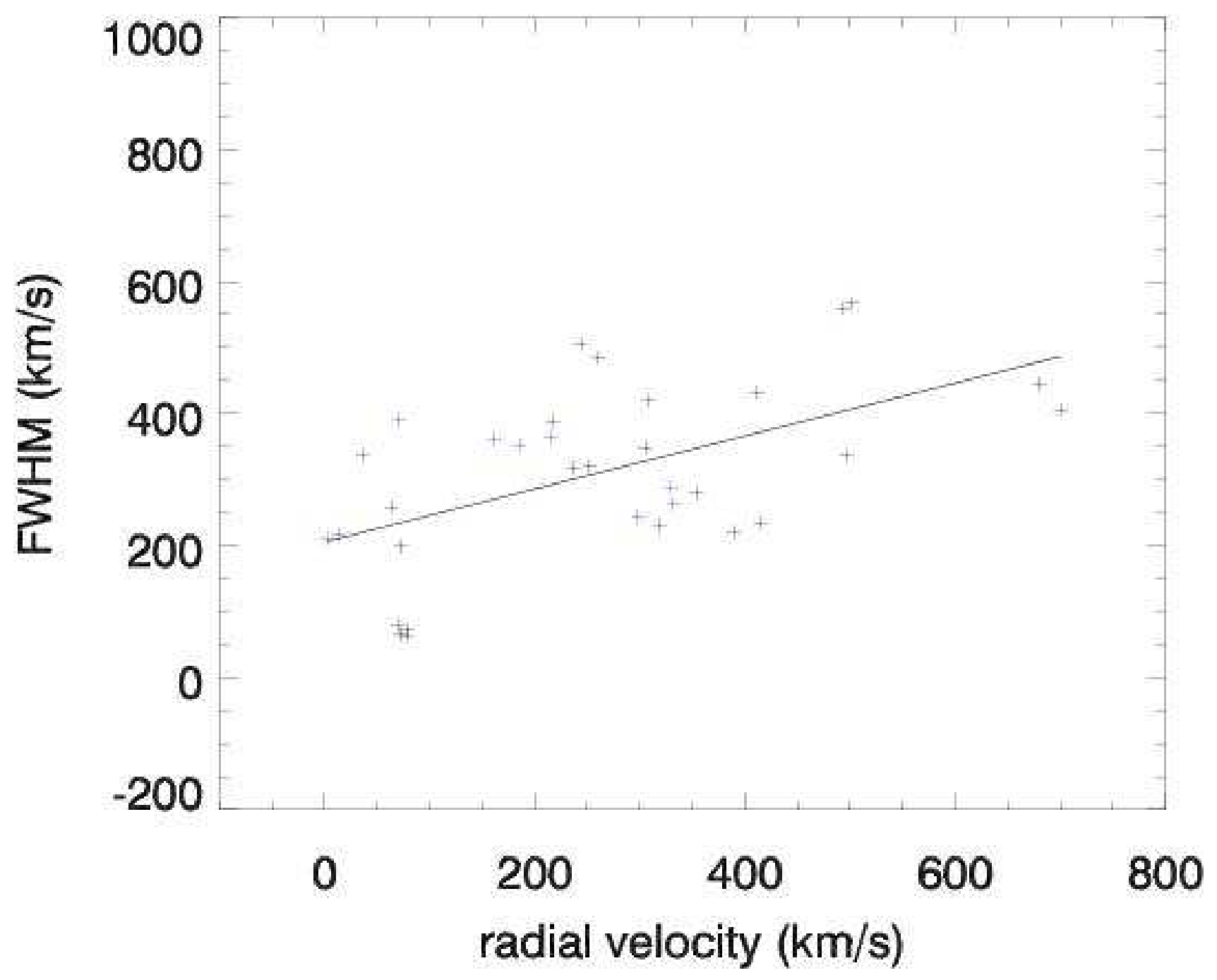

Fig. 11 

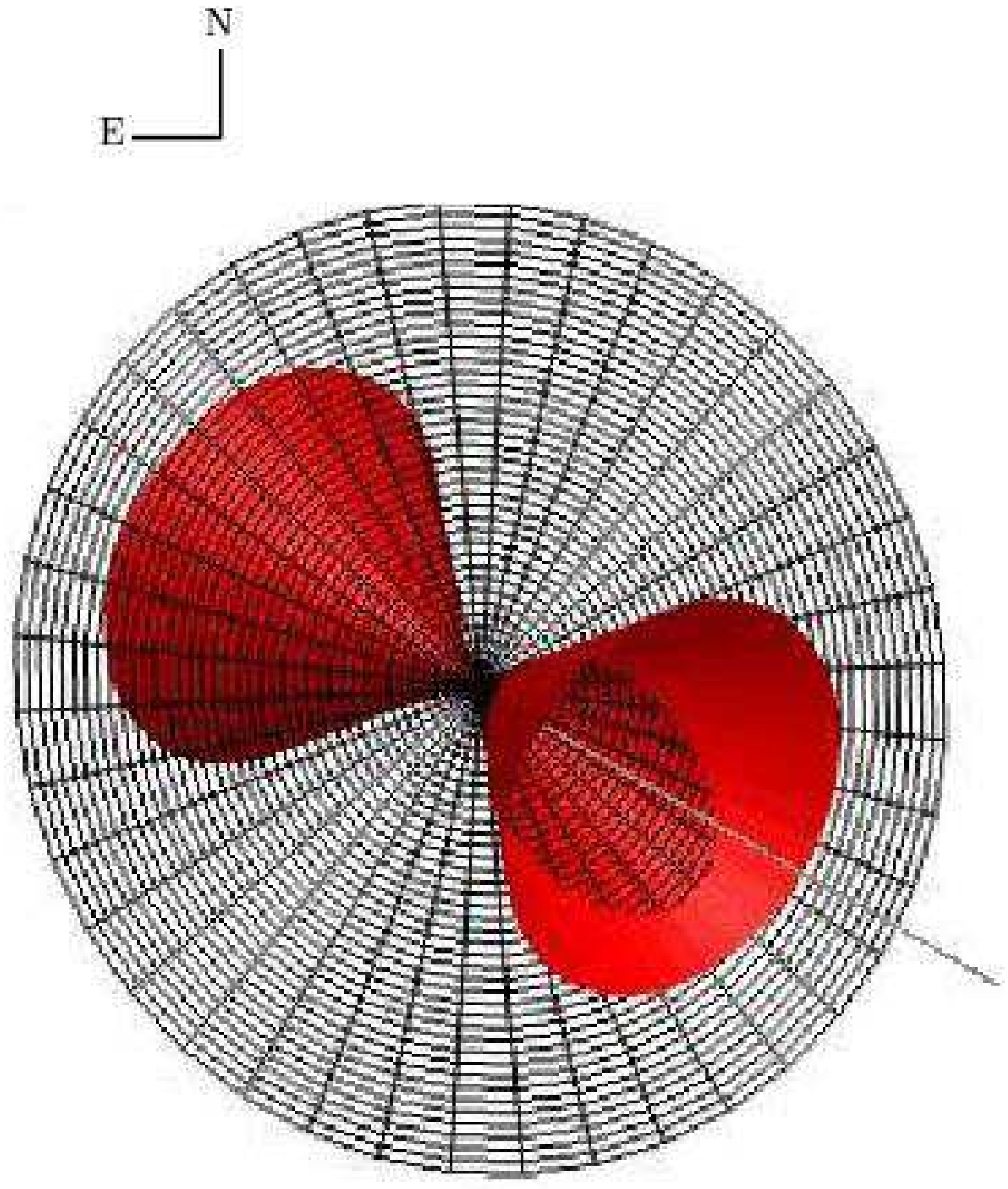

Fig. 12 


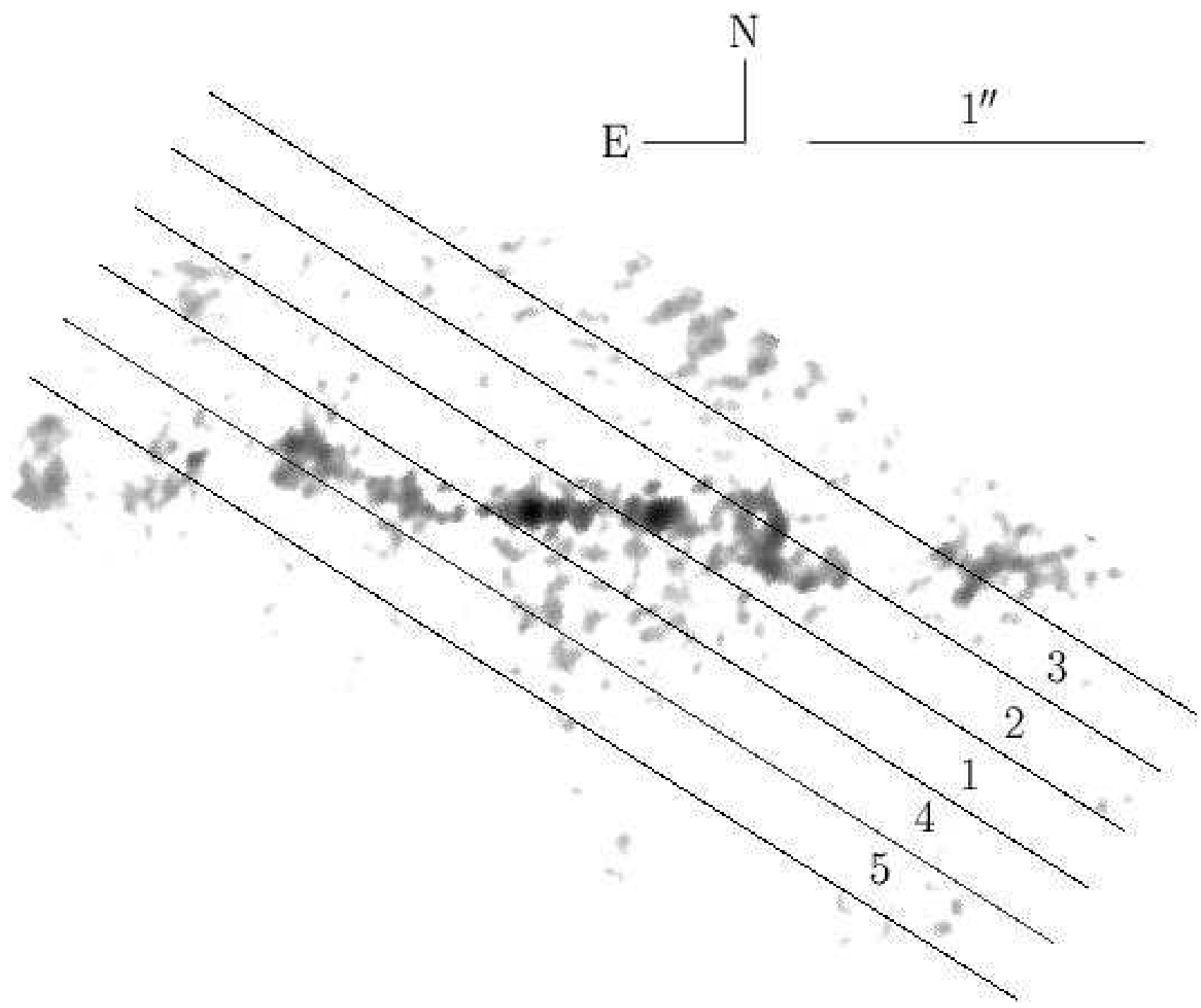

Fig. 13 

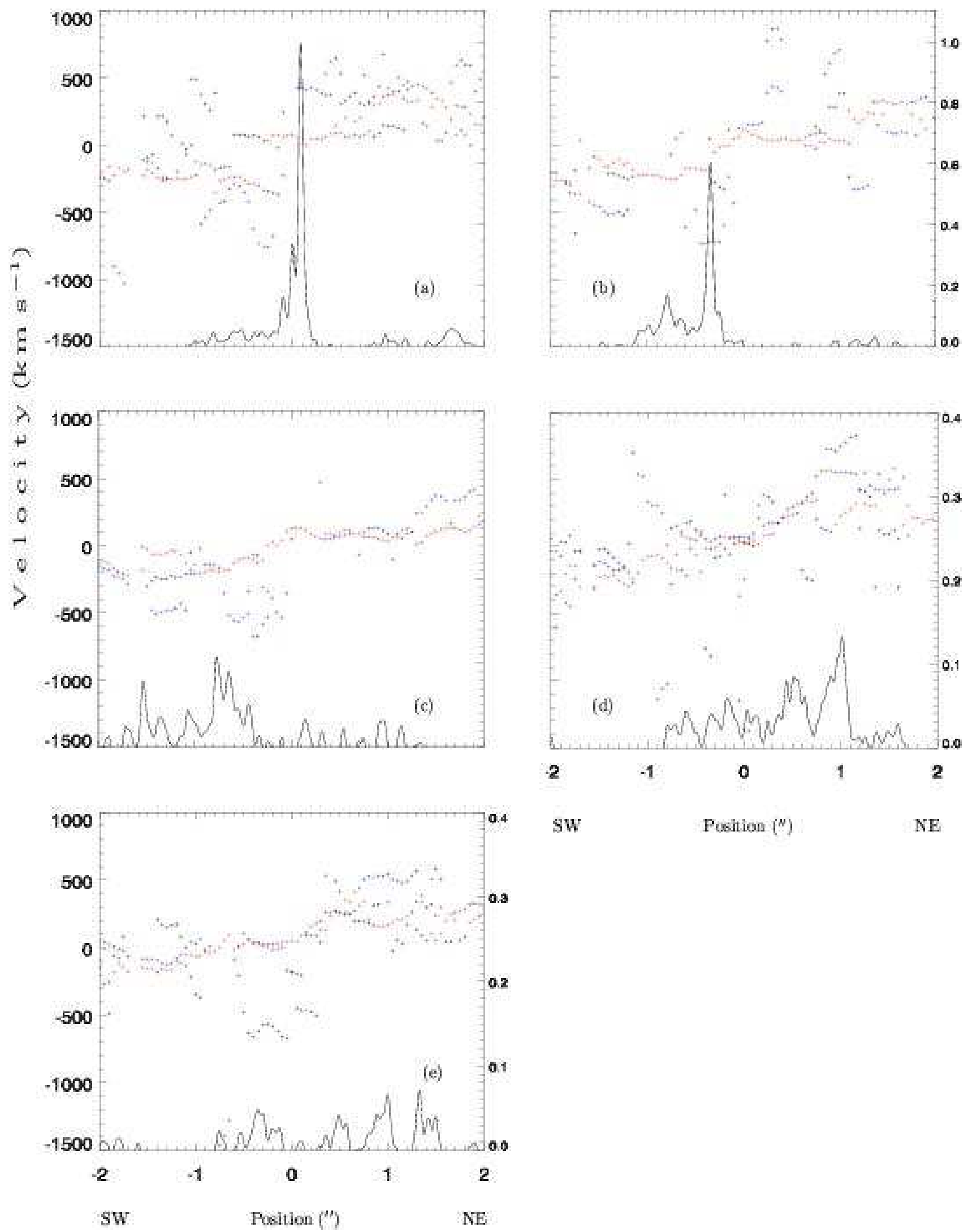

sw

Position ( $(N)$

NE

Fig. 14 

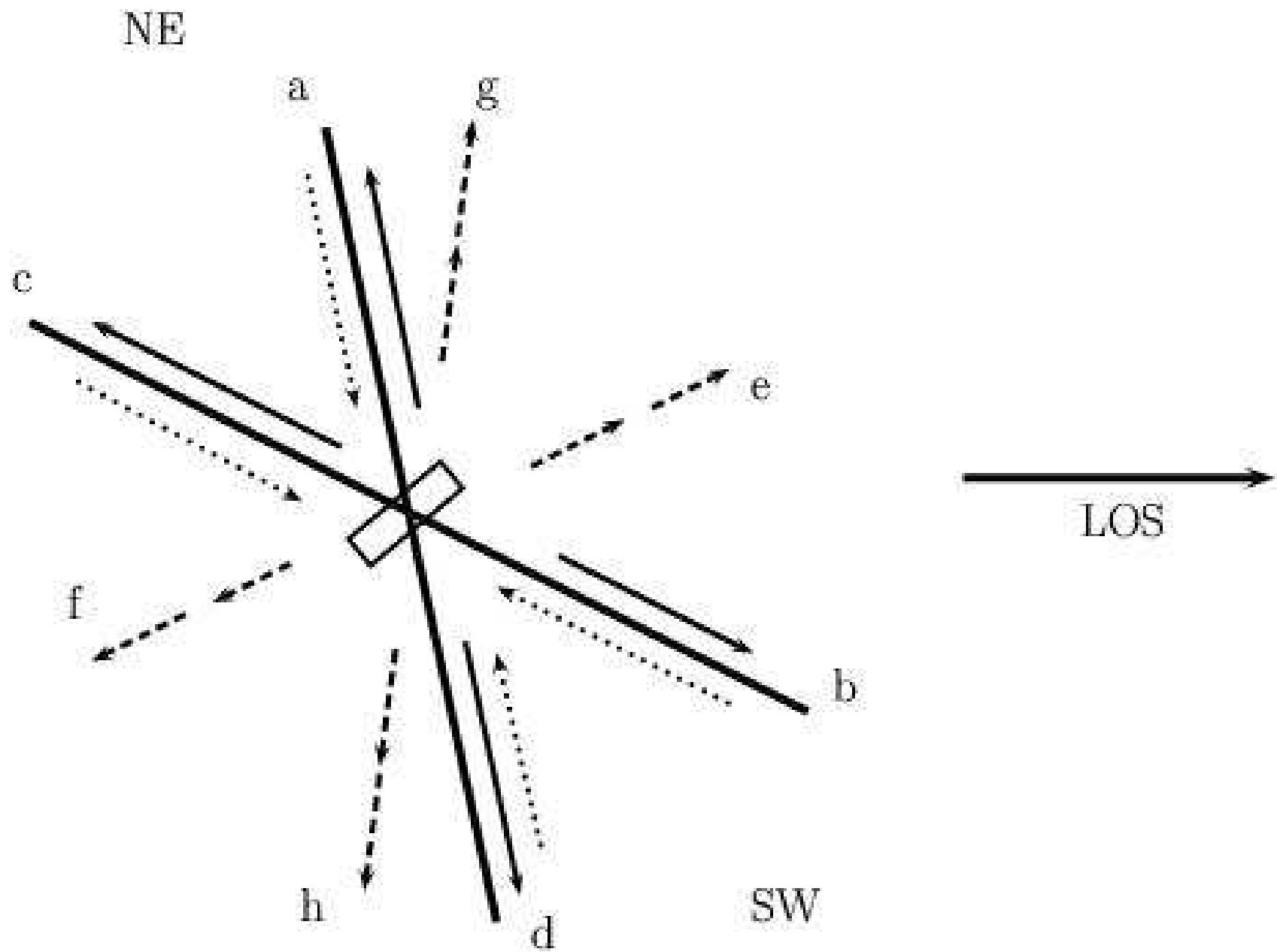

Fig. 15 